\title{
SMU.759 que codifica para una proteasa similar a colagenasa es constitutivo en Streptococcus mutans
}

SMU.759 encoding a collagenase-like protease is constitutive in Streptococcus mutans

SMU.759 que codifica uma protease semelhante à colagenase é constitutiva em Streptococcus mutans

\author{
Eduardo Sáenz-Rivera ${ }^{1}$ \\ Edgar Mauricio Bañol-Acevedo \\ Ramiro Javier Rincón-Rodríguez ${ }^{3}$
}

Recibido: 29 de junio de 2021 Aprobado: 28 de agosto de 2021 Publicado: 16 de septiembre de 2021

Cómo citar este artículo: Saenz-Rivera E, Bañol-Acevedo EM, Rincón-Rodríguez RJ. SMU.759 que codifica para una proteasa similar a colagenasa es constitutivo en Streptococcus mutans. Revista Nacional de Odontología. (2021); 17(1), 1-11. doi: https://doi.org/10.16925/2357-4607.2021.01.04

Artículo de investigación. https://doi.org/10.16925/2357-4607.2021.01.04

1 Facultad de Odontología, Universidad de Antioquia, Medellín, Colombia. Correo electrónico: eduardo.saenz@udea.edu.co

2 Facultad de Odontología, Universidad de Antioquia, Medellín, Colombia.

3 Facultad de Odontología, Universidad de Antioquia, Medellín, Colombia. 


\section{Resumen}

Introducción: en la dentina se encuentra el colágeno tipo I que puede ser degradado por acción enzimática. El gen SMU.759 de Streptococcus mutans, serotipo c, codifica una proteasa con posible actividad colagenasa afectando probablemente este tipo de colágeno y favoreciendo el avance de la caries, dado que, en procesos posteriores a la desmineralización, el colágeno tipo I de la dentina queda expuesto a la acción de esta bacteria. Se pretende determinar si hay diferencias en la presencia o ausencia de SMU.759 en aislados de pacientes con y libres de caries.

Materiales y métodos: se utilizaron aislados de un cepario procedentes de escolares entre 6 y 9 años de edad con y libres de caries de la ciudad de Medellín, Colombia. Se diseñaron los cebadores para SMU.759, se realizó PCR, se observaron los amplificados en un gel de agarosa al $1,2 \%$.

Resultados: SMU.759 se amplificó en todos los aislados utilizados.

Conclusión: el gen SMU.759, que codifica una proteasa, sería un gen constitutivo del genoma de S. mutans.

Palabras clave: SMU.759; colagenasa; colágeno tipo I; dentina, caries.

\section{Abstract}

Introduction: type I collagen is found in dentin, which can be degraded by enzymatic action. The SMU.759 gene of Streptococcus mutans, serotype c, encodes a protease with possible collagenase activity, probably affecting this type of collagen and favoring the progression of caries, since, in processes after demineralization, type I collagen in dentin is exposed to the action of this bacteria. The aim is to determine if there are differences in the presence or absence of SMU.759 in isolates from patients with and free of caries.

Materials and methods: isolates from a strain were used from schoolchildren between 6 and 9 years of age with and free of caries from the city of Medellín, Colombia. The primers for SMU.759 were designed, PCR was performed, the amplified ones were observed on a $1.2 \%$ agarose gel.

Results: SMU.759 was amplified in all the isolates used.

Conclusion: the SMU.759 gene, which encodes a protease, would be a constitutive gene of the S. mutans genome.

Keywords: SMU.759; collagenase; type I collagen; dentin, cavities.

\section{Resumo}

Introdução: o colágeno tipo I é encontrado na dentina, podendo ser degradado por ação enzimática. 0 gene SMU.759 de Streptococcus mutans, sorotipo c, codifica uma protease com possivel atividade colagenase, provavelmente afetando este tipo de colágeno e favorecendo a progressão da cárie, uma vez que, em processos após a desmineralização, o colágeno tipo I na dentina fica exposto ao ação desta bactéria. 0 objetivo é determinar se há diferenças na presença ou ausência de SMU.759 em isolados de pacientes com e sem cárie.

Materiais e métodos: foram utilizados isolados de uma cepa de escolares entre 6 e 9 anos de idade com e sem cárie da cidade de Medellín, Colômbia. Os primers para SMU.759 foram desenhados, a PCR foi realizada e os amplificados foram observados em gel de agarose a 1,2\%.

Resultados: SMU.759 foi amplificado em todos os isolados usados.

Conclusão: o gene SMU.759, que codifica uma protease, seria um gene constitutivo do genoma de S. mutans.

Palavras-chave: SMU.759; colagenase; colágeno tipo l; dentina, cavidades. 


\section{Introducción}

La bacteria Streptococcus mutans (S. mutans) ha sido asociada como uno de los factores etiológicos de la caries. Es gram +, con capacidad para formar biopelículas, catalasa (-), no móvil y productor rápido de ácido láctico. Esta bacteria metaboliza una amplia variedad de carbohidratos y genera ácidos como productos de desecho (1), que le confiere factores de virulencia tales como acidogenicidad, aciduricidad y acidofilicidad (2). Desde la perspectiva del pangenoma, de S. mutans, su pangenoma es abierto, relacionado con un genoma central o core constituido por genes asociados con actividades como el metabolismo y el almacenamiento o procesamiento de información hereditaria (3). En su genoma está presente SMU.759, que cuenta con una longitud de 927 pares de bases (bp) $(2,4)$, se encuentra ubicado en la región 710298 a 711224 bp del genoma (4), y codifica una proteasa compuesta por 308 aminoácidos con una masa molecular calculada de 35,48 kDa (5-7). Este gen se asemeja estructuralmente a homólogos hallados en otras bacterias que presentan acción colagenasa (8). Por otro lado, en la dentina y el cemento se encuentra el colágeno tipo I, II, III, IV, V y VI (9), entre los que se destaca el colágeno tipo I que es sintetizado por el odontoblasto y representa el 90 \% de la matriz extracelular dentinal. Alrededor de este colágeno se depositan los cristales de hidroxiapatita que constituyen la composición inorgánica del diente (9). Estos cristales son disueltos por ácidos orgánicos producidos por la placa bacteriana resultado de su metabolismo a partir de azúcares. Esto genera principalmente ácido láctico, que inicia la pérdida de minerales del esmalte incrementando su porosidad, y permite que la desmineralización progrese subyacente a la superficie dental generando su colapso final por las fuerzas masticatorias (10), y expone la dentina y el colágeno tipo I, el cual sería blanco de colagenasas bacterianas (11). En este proceso carioso interviene el S. mutans, que podría ser uno de los responsables de la degradación orgánica dentinal debido a que contiene el gen SMU.759 con posible expresión de actividad colagenasa (1). Respecto a SMU.759, la información acerca de su actividad proteolítica es ambigua debido a que diversos estudios basados en genes homólogos han encontrado que esta enzima no está involucrada en la degradación del colágeno (12), o está involucrada en generar un aumento en la producción de anticuerpos séricos del huésped aumentando la respuesta inmune (13), o también induce la secreción de citoquinas proinflamatorias en células endoteliales (14), o finalmente está relacionada con la regulación de funciones básicas internas bacterianas (15).

S. mutans está asociado a la producción de caries (10) y posee a SMU.759; probablemente una colagenasa. Por otro lado, en el proceso de caries, el colágeno tipo I es expuesto a la acción bacteriana como producto de la desmineralización dental 
requiriéndose una enzima para degradarlo (11). Hay dudas acerca de la presencia de SMU.759 en el genoma de S. mutans procedentes de niños con caries, al resolverse esta duda, se contribuiría a esclarecer cómo se desarrolla el proceso carioso dental. Este trabajo pretende relacionar la presencia de SMU.759 con la presencia de caries.

Tabla 1. Secuencia del gen SMU.759 obtenida a partir de la base de datos GenBank de National Center for Biotechnology Information (NCBI).

\begin{tabular}{ccccccc}
\hline 1 & atggaaaaaa & ttgttatcac & tgcgactgca & gaatctattg & aacaagttaa & agaattactg \\
\hline 61 & acaagtggtg & ttgaccgtat & ttatgttggt & gagaaagatt & atgcgcttcg & tttaccgcat \\
\hline 121 & gcgtttagct & atgatgactt & aagaaaaatt & gctagcttgg & ttcatgaagc & tggtaaagaa \\
\hline 181 & ttaacggttg & ctgctaatgc & actaatgcat & caagaaatga & tggacaatat & taaaccattt \\
\hline 241 & ttagaattaa & tgaaggaaat & tcaggtagat & tacttagtgg & ttggtgatgc & aggtgttttt \\
\hline 301 & tatgtcaata & agcgtgatgg & ttatcatttt & aaactcattt & atgatacctc & tgttttgtc \\
\hline 361 & acctctagtc & gtcaagttaa & ttttggggc & caacacggtg & cggtagaagc & tgttttggca \\
\hline 421 & cgtgaaattc & cttcggaaga & actgittgaa & atgtccaaaa & atctggaaat & tcctgcagaa \\
\hline 581 & gtcttagttt & acggtgcttc & tgtcattcat & cattccaagc & gacctttaat & acagaattat \\
\hline 601 & tataatttta & ctcacattga & tgatgagaag & acaagagaac & gcggtctgtt & cttatcagaa \\
\hline 661 & ccaaatgatc & ctaaatcgca & ctattctata & tatgaagata & aacacggcac & tcatattttt \\
\hline 721 & atcaataatg & atattgattt & gatgaccaaa & ttgcctgaat & tgattaatca & tcattacaat \\
\hline 781 & cattggaaat & tagatggtat & ctattgtcca & ggacataatt & ttgttgagat & tgttcaactt \\
\hline 841 & ttgttaaag & caagagatat & gatcgaagct & gggactttta & cgcaagatca & ggcttttctt \\
\hline 901 & ttcgatgaac & aaattagaaa & gcttcatcca & gctggtcgtg & gtttagatac & aggattttat \\
\hline
\end{tabular}

Fuente: elaboración propia a partir de National Center for Biotechnology, Streptococcus mutans UA159, Chromosome, 2015 (6).

\section{Materiales y métodos}

Muestras y obtención de ADN: Se utilizaron 25 aislados procedentes de muestras de lesiones cavitadas y 25 aislados de lesiones de mancha blanca, procedentes de niños con caries, y 25 aislados de placa bacteriana procedentes de niños sin caries, los cuales pertenecen al cepario del laboratorio de biología celular y molecular de los profesores Mónica Parada y Ramiro Rincón, de la Facultad de Odontología de la Universidad de Antioquia. Estos se obtuvieron de muestras procedentes de escolares entre 6 y 9 años de edad con y libres de caries de la Institución Educativa Javiera Londoño de la ciudad de Medellín-Colombia. Para hacer la extracción del ADN se utilizó el protocolo estandarizado en este laboratorio. Los aislados se sembraron en medio líquido $\mathrm{BHI}$ por 48 horas a $37^{\circ} \mathrm{C}$, para obtener el concentrado de bacterias se 
centrifugó a 3000 rpm, se eliminó el sobrenadante y se lavó con agua milli-Q, y posteriormente, se adiciona $200 \mu \mathrm{L}$ de TE. Se hierve por 10 minutos a $100^{\circ} \mathrm{C}$, se centrifuga a 21000 rpm y se coloca en otro tubo el sobrenadante, el cual se congeló a $-80^{\circ} \mathrm{C}$.

Diseño de primers y amplificación de SMU.759 por PCR: Se utilizó el software Primer3 (http://bioinfo.ut.ee/primer3-0.4.0) para diseñar los cebadores para la amplificación: SMU.759 forward: 5' - AAT TGT TAT CAC TGC GAC TGC - 3', SMU.759 reverse: $5^{\prime}$ - TTG TCC ATC ATT TCT TGA TGC - 3'. Las condiciones para el PCR fueron: temperatura de denaturación inicial a $95^{\circ}$ por 2 minutos, 30 ciclos; denaturación a $95^{\circ}$ por 30 segundos, aneliación a $60^{\circ}$, extensión a $72^{\circ}$ por 1 minuto, y una extensión final a $72^{\circ}$ por 7 minutos. El volumen final de la reacción fue de $25 \mu \mathrm{L}$. En un gel de agarosa al 1,2 \% se observaron los amplificados utilizando una cámara de electroforesis (Labnet@) y un transiluminador de luz ultravioleta (select®).

\section{Resultados}

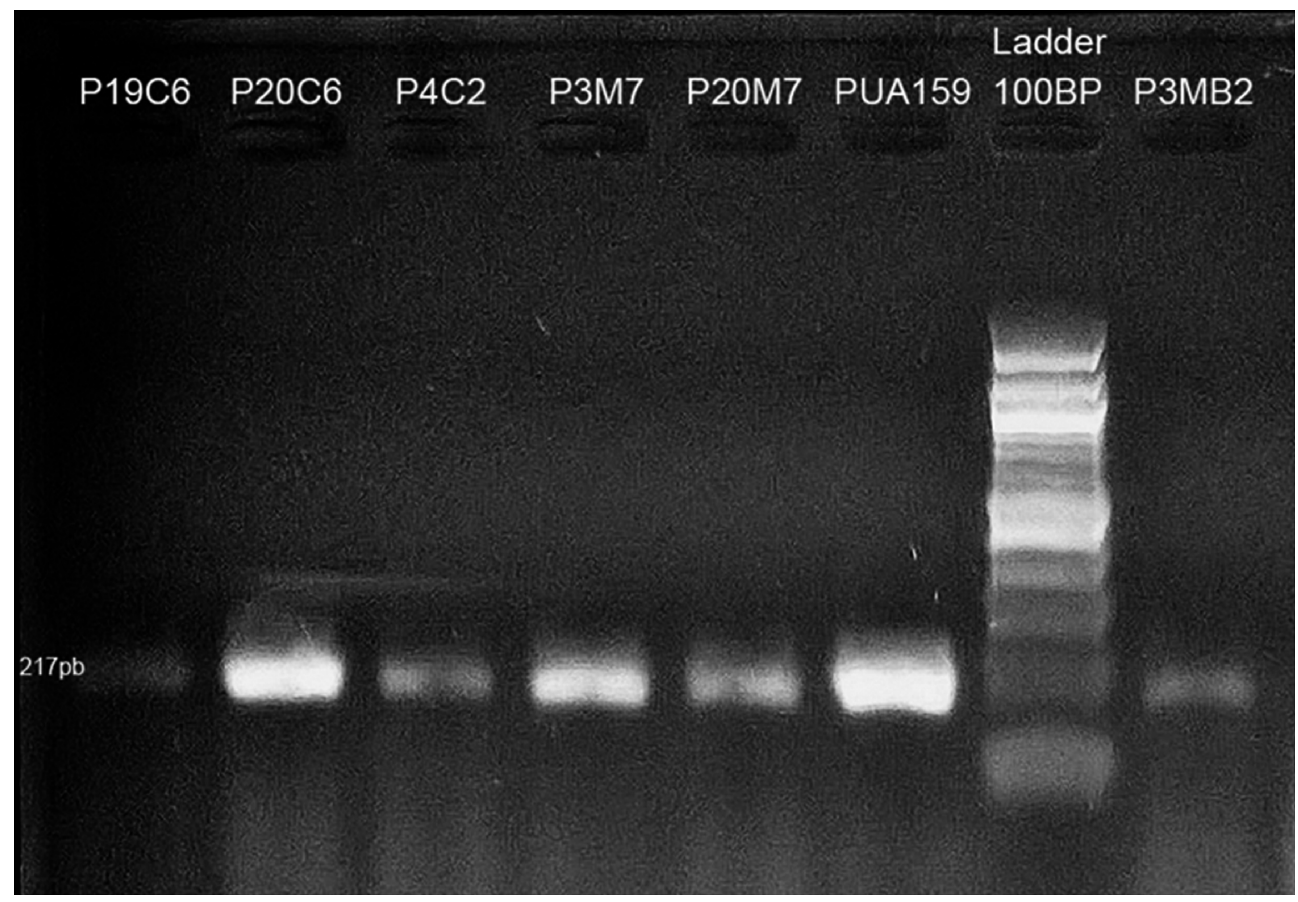

Figura 1. Fotografía de SMU.759 en los aislados. Amplificado de los pacientes. 19, 20, 4,3. C: material reblandecido de lesión cavitada procedentes de niños con caries. M: biopelícula que cubría lesión de mancha blancas procedentes de niños con caries. MB: biopelícula procedente de niños sin caries. PUA159: cepa de referencia S. mutans UA159. Tamaño del amplificado: $217 \mathrm{pb}$.

Fuente: elaboración propia. 
Se encontró que SMU.759 se amplificó tanto en los aislados procedentes de pacientes con y sin caries, lo cual nos indica que no hay diferencias entre los dos grupos.

\section{Discusión}

Este trabajo pretendió encontrar la existencia de diferencias en la presencia de SMU.759 en los aislados procedentes de pacientes con y sin caries para relacionar este gen con el proceso carioso, específicamente en la degradación del colágeno tipo I que está presente en la dentina. Los resultados encontrados muestran que SMU.759 se amplificó en todos los aislados, sin obtener diferencias entre los grupos, lo que permite inferir que probablemente SMU.759 sea un gen constitutivo.

Los genes constitutivos son esenciales para la supervivencia, fueron adquiridos durante la evolución y se mantienen como una dotación de genes para actuar dependiendo del sitio, substrato y momento fisiopatológico, lo que permite al organismo relacionarse con el ambiente, evitar la predación o interactuar con el hospedero. Son genes requeridos para el mantenimiento de funciones celulares esenciales y se expresan bajo condiciones normales, independientemente del tipo de ambiente, estado de desarrollo, estado del ciclo celular o señales externas (16). Søborg et al. (17), en un análisis In silico, compararon mediante homología, genes asociados a virulencia en patógenos bacterianos humanos (toxinas, adhesinas, sistemas de secreción, reguladores, genes relacionados con la inflamación y de resistencia involucrados en infecciones del tracto urinario, enfermedades gastrointestinales, infecciones de la piel y cuerpos extraños e infecciones sistémicas) en diferentes nichos ecológicos para comprender el papel de los entornos naturales en la evolución de la virulencia bacteriana. Los nichos se caracterizaron por condiciones ambientales altamente diferentes en términos de $\mathrm{pH}$, presión, temperatura, salinidad y disponibilidad de nutrientes. En total se analizaron 24 genes de virulencia bacteriana en 46 conjuntos de datos metagenómicos ambientales diversos, que representaron diversos suelos, agua de mar, agua dulce, sedimentos marinos, aguas termales, aguas profundas, esteras hipersalinas, microbialitas, gusanos sin intestinos y hielo glacial. Encontraron ubicuidad global de 16 genes homólogos de virulencia humana bacteriana involucrados en infecciones del tracto urinario, enfermedades gastrointestinales, enfermedades de la piel e infecciones de heridas y sistémicas (17). También observaron más del $95 \%$ de homología completa de varios genes de virulencia, las secuencias traducidas de los genes ambientales y clínicos fueron idénticas entre un 50-100 \%. En cuanto a los análisis filogenéticos, encontraron posiciones de ramificación profunda de algunos de los 
homólogos genéticos ambientales, lo que sugiere que representan linajes antiguos en la filogenia de los genes clínicos. Además, 15 homólogos de genes de virulencia se detectaron en metatranscriptomas, lo que proporciona evidencia de la expresión ambiental. Por otro lado, los homólogos de genes de virulencia se distribuyeron globalmente en seis continentes y en cuatro océanos, es decir, en todos los continentes y océanos para los que había metagenomas relevantes disponibles.

Estos hallazgos apuntaron a un importante papel ecológico de los genes para la actividad y supervivencia de las bacterias ambientales y el alto grado de conservación de la secuencia entre varios de los genes ambientales y clínicos sugiriendo orígenes ancestrales comunes (17). La presencia ubicua de los genes sugiere que tienen funciones importantes, y se ha propuesto que son genes de mantenimiento esenciales para la supervivencia y la proliferación en entornos externos (18). Por otro lado, basados en estudios de pangenoma (19), específicamente para S. mutans (3), permite obtener y estudiar muchos genomas diferentes de la misma especie, lo cual proporciona suficientes datos para realizar comparaciones tanto de su genoma central como de su genoma no esencial o prescindible (20). Es así como Meng et al. (3), concluyeron en un análisis pangenómico de 183 aislados de S. mutans, que el genoma de esta bacteria es pangenoma abierto, de donde se deriva que nuevos genes se encontrarán a medida que se secuencian más genomas. Igualmente, S. mutans presenta un genoma core constituido por genes relacionados con actividades vitales dentro de la bacteria, como el metabolismo y el almacenamiento o procesamiento de información hereditaria, que ocupa el 35,6 y el 26,6 \% de los genes core, respectivamente.

Inicialmente, se consideró que la presencia de SMU.759 era necesaria en la expresión de la fisiopatología de S. mutans cuando se encuentra en los micronichos, ya sea en la biopelícula o posterior a la destrucción del esmalte por generación de ácidos, quedando expuesta la dentina e induciendo la expresión de esta proteasa. Kato, Takahashi y Kuramitsu (8) evaluaron la función de una proteasa homóloga a SMU.759 en la cepa ATCC 53977 de Porphyromonas gingivalis, bacteria asociada a la enfermedad periodontal (21), y encontraron que podía degradar colágeno tipo | fibrilar soluble o reconstituido, azocoll (sustrato de colágeno sintético) y colágeno tipo I desnaturalizado con calor. Sin embargo, no hidrolizaba gelatina, ni el sustrato sintético PZ-PLGPA (4-fenilazobencil-oxicarbonil-ProLeu-Gli-Pro-D-Arg), como sí lo hacen otras colagenasas bacterianas (22), lo que sugiere que ésta proteasa puede tener actividad colagenasa. Por otro lado, Kuramitsu, Yoneda y Madden (12), evaluaron la actividad colagenasa, en esta misma bacteria, en una cepa mutante sin el gen prtC (PrtC-), encontrando que esta cepa presentaba una fuerte actividad enzimática aún sin la proteasa codificada, sugiriendo así que esta enzima no es la mayor responsable 
en la degradación del colágeno por parte de esta bacteria periodontopatógena, lo que indicaría que este gen presente en $P$. gingivalis expresa actividad colagenasa pero podría no ser la principal responsable en la degradación del colágeno tipo I, en este caso, en el micronicho periodontal. En contraste, Lamont y Jenkinson (15), identificaron proteasas homólogas a SMU.759 en Methanobacterium, Methanococcus jannaschii, Bacillus subtilis o Haemophilus influenzae, las dos primeras fueron encontradas en ambientes termales, y las dos segundas, bacterias comensales en el ser humano, y se encontró similitud estructural con este grupo de colagenasas. Sin embargo, estas bacterias no presentaron actividad colagenolítica, por lo que se sugirió que la presencia de esta enzima podría estar relacionada con la regulación de otras funciones básicas internas, o en el caso de Bacillus subtilis o Haemophilus influenzae, su expresión podría no desencadenarse debido a la ausencia de colágeno expuesto en el medio en el que se encuentran, en el intestino (23) y en las vías aéreas superiores (24) respectivamente.

La información sobre este gen también es ambigua debido a que no se ha definido exactamente su función. Como se ha descrito previamente, algunos lo relacionan con la degradación enzimática, y otros afirman que induce en el huésped reacciones inflamatorias e inmunológicas, como Beikler et al (13), que encontraron que la presencia de esta enzima estaba relacionada con respuestas en la producción de anticuerpos séricos del hospedador. Estos hallazgos fueron encontrados al evaluar una muestra obtenida de 34 pacientes que presentaban enfermedad periodontal; las inmunoglobulinas producidas ante el reconocimiento de la enzima fueron específicamente: IgA en un $97 \%$ de la muestra estudiada, e IgG en un $100 \%$ con una distribución de 65 \% para su subdivisión IgG2, 47 \% para IgG3, 38 \% para IgG1 y 29 \% para IgG4 (13). En otro escenario, Wu et al. (14) encontraron que esta enzima induce la secreción de citoquinas inflamatorias tales como IL-1a, IL-8, y TNF-a por parte de las células endoteliales. Se toma en consideración que a pesar de que esta enzima pudiera ser una colagenasa, su efecto en la respuesta inflamatoria podría potenciar la actividad enzimática de otras colagenasas presentes en las células del huésped, tales como las metaloproteinasas 1 y 8 (MMP-1 y MMP-8) presentes en la matriz extracelular de la dentina (25-27), lo cual podría provocar una acción sinérgica en el avance de las lesiones cariosas en las que S. mutans estaría presente. Surge la necesidad de evaluar esta posible actividad sinérgica, de tal manera que adicional a que SMU.759 podría estar relacionada de manera directa en el proceso carioso mediante su expresión colagenasa, también podría actuar como un cofactor en su progresión al potenciar otras colagenasas presentes en la dentina. 
Estos hallazgos ponen de manifiesto que la presencia de esta proteasa en diferentes bacterias no ha sido relacionada de manera directa con una función específica, pero se sugiere que puede tener actividad colagenasa cuando la bacteria está en un micronicho con sustrato de colágeno.

Este estudio concluye que SMU.759 es constitutivo de S. mutans. Su expresión dependerá de la fisiopatología para degradar el colágeno o realizar otra función dependiendo del micronicho en donde se encuentre. Probablemente en el proceso carioso, S. mutans al contacto con la dentina es inducida a expresar este gen para degradar el colágeno tipo I.

\section{Agradecimientos}

Agradecemos a la Facultad de Odontología de la Universidad de Antioquia y al laboratorio de biología celular y molecular de los profesores Mónica Parada y Ramiro Rincón por la colaboración y orientación prestada.

\section{Conflicto de intereses}

Los autores afirman que no tienen conflicto de intereses.

\section{Referencias}

1. Ajdić D, McShan WM, McLaughlin RE, Savić G, Chang J, Carson MB, et al. Genome sequence of Streptococcus mutans UA159, a cariogenic dental pathogen. Proc Natl Acad Sci U S A. 2002;99(22):14434-9.

2. Núñez DP, García L. Bioquímica de la caries dental. Rev Haban Cienc Méd. 2010;9(2):156-166.

3. Meng P, Lu C, Zhang Q, Lin J, Chen F. Exploring the Genomic Diversity and Cariogenic Differences of Streptococcus mutans Strains Through Pan-Genome and Comparative Genome Analysis. Curr Microbiol. 2017;74(10):1200-1209. doi: 10.1007/s00284-017-1305-z

4. National Center for Biotechnology Information. Bethesda: National Center for Biotechnology Information. Streptococcus mutans UA159 chromosome, complete genome. Disponible en: https://www.ncbi.nlm.nih.gov/nuccore/NC_004350.2?report=genbank\&from=710298\& to $=711224$. [Consultado 20 Nov 2020]. 
5. ExPASy Bioinformatics Resource Portal [Internet]. Lausanne, Switzerland: Swiss Institute of Bioinformatics. Compute pl/Mw tool. Disponible en: http://web.expasy.org/compute_pi/ [Consultado 20 Nov 2020].

6. National Center for Biotechnology Information. Bethesda: National Center for Biotechnology Information. SMU_759 protease [Streptococcus mutans UA159]. Disponible en: http://www. ncbi.nlm.nih.gov/gene?term=SMU.759 [Consultado 20 Nov 2020].

7. National Center for Biotechnology Information. Bethesda: National Center for Biotechnology Information. Protease [Streptococcus mutans UA159]. Disponible en: https://www.ncbi.nlm. nih.gov/protein/NP_721176.1?report=genpept [Consultado 20 Nov 2020].

8. Kato T, Takahashi N, Kuramitsu HK. Sequence analysis and characterization of the Porphyromonas gingivalis prtC gene, which expresses a novel collagenase activity. J Bacteriol.1992; 174(12):3889-95.

9. Butler WT. Dentin matrix proteins. Eur J Oral Sci. 1998; 106(1):204-210.

10. Ojeda JC, Oviedo E, Salas LA. Streptococcus mutans y caries dental. CES Odontol. 2013;26(1):44-56.

11. López M, Amaral S, Bussadori S. Proteolisis enzimática del colágeno dentinario. Odontoestomatología. 2010;12(14):35-44.

12. Kuramitsu HK, Yoneda M, Madden T. Proteases and Collagenases of Porphyromonas Gingivalis. ADR. 1995;9(1):37-40.

13. Beikler T, Ehmke B, Wittstock M, Schmidt H, Karch H, Flemmig TF. Serum antibody reactivity against recombinant PrtC of Porphyromonas gingivalis following periodontal therapy. $J$ Periodontal Res. 2003;38(3):276-81.

14. Wu YM, Chen LL, Yan J, Zhuang CY, Gu ZY. Effect of Porphyromonas gingivalis PrtC on cytokine expression in ECV304 endothelial cells and its level in subgingival plaques from patients with chronic periodontitis. Acta Pharmacol Sin. 2007;28(7):1015-23.

15. LamontRJ, Jenkinson HF. Life below the gum line: pathogenic mechanisms of Porphyromonas gingivalis. Microbiol Mol Biol Rev. 1998;62(4):1244-63.

16. Eisenberg E, Levanon EY. Human housekeeping genes, revisited. Trends Genet. 2013; 29(10):569-74. 
17. Søborg DA, Hendriksen NB, Kilian M, Christensen JH, Kroer N. Bacterial Human Virulence Genes across Diverse Habitats As Assessed by In silico Analysis of Environmental Metagenomes. Front. Microbiol. 2016;7:1712.

18. Søborg, DA, Hendriksen NB, Kilian M,Kroer N. Widespread occurrence of bacterial human virulence determinants in soil and freshwater environments. Appl. Environ. Microbiol. 2013;79;5488-5497.

19. Rouli L, Mrhej V, Fournier PE, Raoult D. The bacterial pangenome as a new tool for analysing pathogenic bacteria. New Microbe and New Infect. 2015;7:72-85 doi: 10.1016/j. nmni.2015.06.005

20. Bedoya-Correa CM, Rincón RRJ, Parada-Sanchez MT. Genomic and phenotypic of Streptococcus mutans. Journal of Oral Biosciences. 2018;15;61(1):22-31. doi: 10.1016/j. job.2018.11.001

21. Rafiei 1, Kiani F, Sayehmiri F, Sayehmiri K, Sheikhi A, Zamanian Azodi M. Study of Porphyromonas gingivalis in periodontal diseases: A systematic review and meta-analysis. Med J Islam Repub Iran. 2017;12;31:62.

22. Harrington DJ. Bacterial collagenases and collagen-degrading enzymes and their potential role in human disease. Infect Immun. 1996;64(6):1885-91.

23. Rhayat L, Maresca M, Nicoletti C, et al. Effect of Bacillus subtilis Strains on Intestinal Barrier Function and Inflammatory Response. Front Immunol. 2019;10:564.

24. King P. Haemophilus influenzae and the lung (Haemophilus and the lung). Clin Trans/ Med. 2012;1(1):10.

25. Klein T, Bischoff R. Physiology and pathophysiology of matrix metalloproteases. Amino Acids. 2011;41(2):271-290.

26. Sapna G, Gokul S, Bagri-Manjrekar K. Matrix metalloproteinases and periodontal diseases. Oral Dis. 2014;20(6):538-50.

27. Pereira V, Asquino N, Apellaniz D, Bueno Luis, Tapia G, Bologna R. Metaloproteinasas de la matriz extracelular (mmps) en Odontología. Odontoestomatología. 2016; 18(28): 20-29. 